\title{
Avaliação de uma Dinâmica Vivencial para o Ensino de Gerenciamento de Projetos em Cursos de Computação
}

\author{
Giani Petri $^{1,2}$, Christiane Gresse von Wangenheim ${ }^{2}$, Bruno B. Boniati ${ }^{3}$, \\ Alex R. Weber', 4 \\ ${ }^{1}$ Universidade Federal de Santa Maria (UFSM) \\ Av. Roraima, 1000 - 97.105-900 - Santa Maria, RS - Brasil \\ ${ }^{2}$ Programa de Pós-Graduação em Ciência da Computação (PPGCC) \\ Universidade Federal de Santa Catarina (UFSC) - Florianópolis, SC - Brasil \\ ${ }^{3}$ Instituto Federal de Educação, Ciência e Tecnologia Farroupilha (IFFar) \\ Campus Frederico Westphalen - Frederico Westphalen, RS - Brasil \\ ${ }^{4}$ Instituto Federal de Educação, Ciência e Tecnologia de Santa Catarina (IFSC) \\ Campus Xanxerê - Xanxerê, SC - Brasil \\ gpetri@inf.ufsm.br, c.wangenheimeufsc.br, bruno.boniati@iffarroupilha.edu.br, \\ alex. weberdifsc.edu.br
}

\begin{abstract}
The current scenario in the software industry requires qualified professionals who know the project management process. In this context, experiential dynamics, used in professional workshops, are being used in the academic context for project management education. Thus, this article aims to evaluate the quality, in terms of perceived learning and player experience, of PMDome, an instructional strategy used to simulate the planning and execution phases of project management in computing courses. The evaluation from 47 students from different educational levels provides evidence on the benefits of PMDome for students' learning, as well as its positive contribution to the social interaction, fun, satisfaction and confidence.
\end{abstract}

Resumo. $O$ cenário atual do setor de software requer profissionais qualificados e que conheçam os processos de gerenciamento de projetos. Neste contexto, dinâmicas vivenciais e de simulação, utilizadas em workshops profissionais, estão sendo utilizadas no contexto acadêmico para o ensino de gerenciamento de projetos. Assim, este trabalho objetiva avaliar a qualidade, em termos de percepção da aprendizagem e experiência do jogador, da dinâmica PMDome, uma estratégia de ensino usada para simular as fases de planejamento e execução do gerenciamento de projetos em cursos de computação. A avaliação, envolvendo 47 alunos de cursos de diferentes níveis de ensino, evidenciam a qualidade da dinâmica PMDome para a aprendizagem dos alunos, além de contribuir positivamente para a interação social, diversão, satisfação e confiança.

\section{Introdução}

As estatísticas do cenário mundial de software demonstram que a maioria dos projetos de software ainda apresentam problemas [Standish Group, 2015]. Conforme o Chaos 
Report de 2015, cerca de 52\% dos projetos precisam de alteração, $19 \%$ falham por completo e, apenas, $29 \%$ são completados de forma satisfatória, respeitando o prazo e o orçamento planejado [Standish Group, 2015]. Um dos motivos para estes resultados, dentre outros, é a falta de conhecimento, da equipe de desenvolvimento, nos processos de gerenciamento de projetos [Standish Group, 2015].

Nos cursos de computação, a gerência de projetos é ensinada em disciplinas específicas ou como um tópico menor em disciplinas de Engenharia de Software [ACM/IEEE-CS, 2013]. Tipicamente a gerência de projetos é ensinada por meio de aulas expositivas, que são adequadas para apresentar informações e conceitos [Barnes et al., 2008]. Mas, por ser uma estratégia centrada no professor, não apoia a aplicação prática de competências [Barnes et al., 2008; Parsons, 2011]. No entanto, considerando o perfil dos estudantes de computação atualmente [Parsons, 2011], é necessário utilizar estratégias de ensino focadas no aluno, permitindo que eles aprendam fazendo, por meio de suas próprias experiências.

Atualmente, há diversos trabalhos que objetivam complementar o ensino de gerenciamento de projetos por meio de atividades práticas, que proporcione aos alunos uma aprendizagem ativa [Calderón e Ruiz, 2015; Letra et al., 2015; Petri et al., 2017a]. Jogos educacionais estão sendo utilizados como estratégia instrucional para revisar conceitos básicos por meio de quiz games [Petri et al., 2016], simular o ciclo de vida de um projeto de software em um jogo digital [Calderón e Ruiz, 2013], ou praticar os conceitos de Scrum no gerenciamento ágil de projetos [Battistella et al., 2016]. Além disso, dinâmicas que objetivam simular um projeto envolvendo toda equipe no desenvolvimento de algum produto, também estão sendo utilizadas como estratégia instrucional [Gresse von Wangenheim et al., 2013; Paasivaara et al., 2014]. Nos últimos anos, o uso de dinâmicas vivenciais e de simulação, que são utilizadas em workshops profissionais, específicos para a formação de gerentes de projetos, também estão sendo utilizadas no contexto acadêmico [Gresse von Wangenheim et al., 2013, Petri e Marcon Jr, 2014].

Deste modo, acredita-se que os jogos e as dinâmicas vivenciais possam ser estratégias instrucionais eficazes e eficientes para o ensino de gerenciamento de projetos [Gibson e Bell, 2013; Souza e França, 2016; Kosa et al., 2016]. No entanto, estas alegações são questionáveis ou não rigorosamente comprovadas [Calderón e Ruiz, 2015; Petri e Gresse von Wangenheim, 2017]. Na prática, as dinâmicas vivenciais utilizadas para o ensino de gerenciamento de projetos em contexto educacional necessitam mostrar o impacto esperado de aprendizagem e/ou o engajamento que elas prometem [Connolly et al., 2012; Kosa et al., 2016; Çiftci, 2018]. Portanto, é essencial avaliar sistematicamente a qualidade dessas dinâmicas, a fim de obter provas sólidas sobre a sua qualidade [Kosa et al., 2016].

Diante disso, este trabalho objetiva avaliar a qualidade de uma dinâmica vivencial utilizada em workshops profissionais, conhecida como PMDome [PMDome, 2017], como uma estratégia de ensino de gerenciamento de projetos. A dinâmica PMDome objetiva que os participantes assimilem os principais conceitos do gerenciamento de projetos, reconheçam a importância do planejamento e desenvolvam um produto durante a atividade. O produto final do projeto é uma estrutura física denominada Domo Geodésico, construída a partir de folhas de papel e fita adesiva. A escolha da dinâmica é justificada devido a sua aderência aos objetivos de aprendizagem 
e perfil dos alunos participantes. Para a avaliação da qualidade da dinâmica PMDome é utilizado o modelo MEEGA+ [Petri et al., 2017b], um modelo sistematicamente desenvolvido para a avaliação de jogos educacionais, que avalia a qualidade do jogo em termos de experiência do jogador e percepção da aprendizagem. A avaliação é realizada por uma série de estudos de caso envolvendo um total de 47 alunos de computação de diferentes níveis de ensino.

\section{Método de Pesquisa}

Com o objetivo de avaliar a qualidade da dinâmica PMDome em termos de experiência do jogador e percepção da aprendizagem do ponto de vista de alunos de computação, foi realizada uma série de estudos de caso [Yin, 2017], como ilustrado na Figura 1.

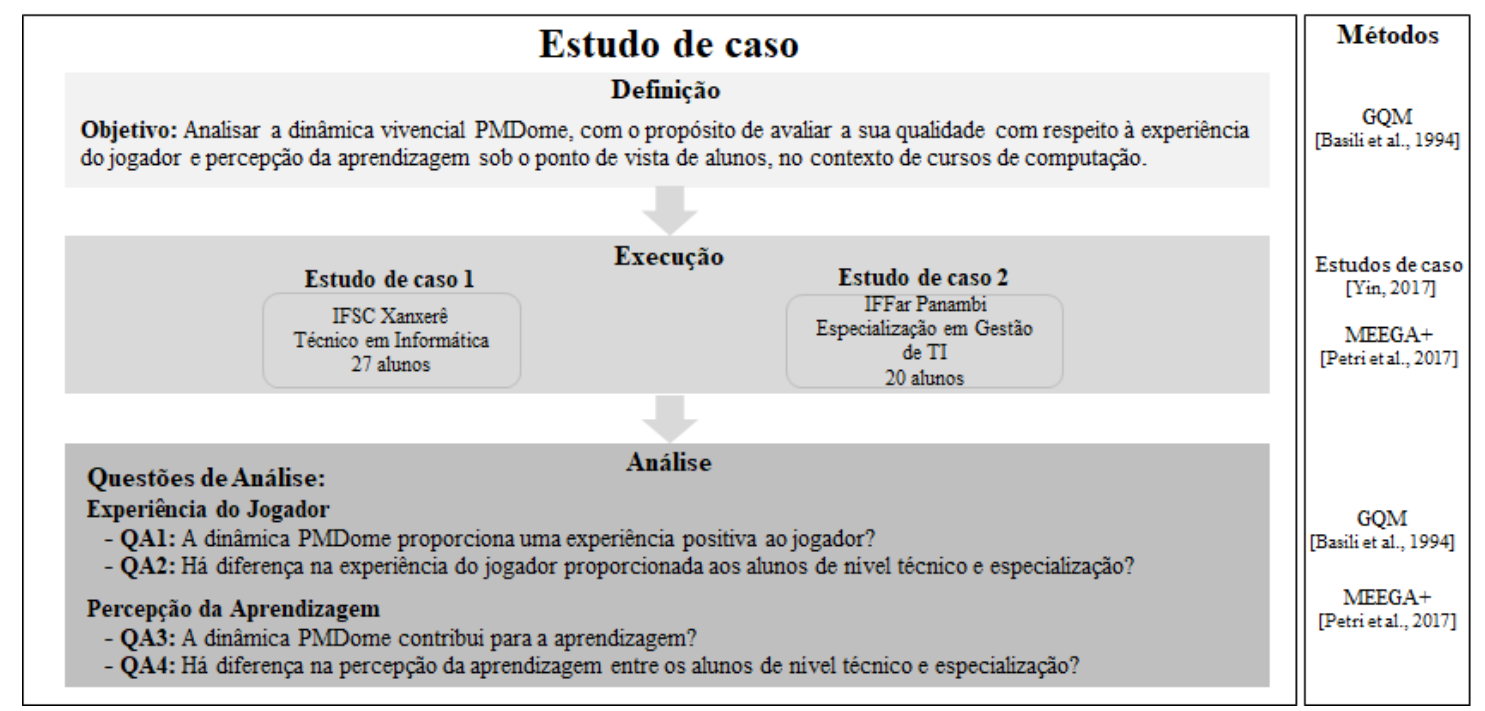

Figura 1. Método de pesquisa

Seguindo a abordagem GQM (Goal/Question/Metric) [Basili et al., 1994], na fase de definição, o objetivo do trabalho é decomposto em aspectos de qualidade e questões de análise para serem analisadas com base no modelo MEEGA+ [Petri et al., 2017b]. Na fase de execução, os estudos de caso são planejados e executados com o público-alvo. Após o tratamento (aplicação da dinâmica PMDome), o questionário do modelo MEEGA+, adaptado para o contexto da dinâmica, é respondido pelos participantes (alunos) para coletar os dados da avaliação da dinâmica PMDome. Na fase de análise, os dados coletados nos dois estudos de caso são agrupados para a análise da qualidade da dinâmica PMDome, e então, responder as questões de análise definidas. Os dados foram analisados seguindo o modelo MEEGA+ utilizando técnicas de estatística descritiva, em termos de distribuição da frequência e medida da tendência central (mediana), de modo a responder as questões de análise. A partir dos dados coletados, os percentuais das frequências das respostas foram identificados.

\section{A Dinâmica PMDome}

A dinâmica PMDome tem como objetivo de aprendizagem transmitir, dentro do contexto de gerenciamento de projetos, a importância do planejamento e o impacto que a falta do mesmo pode ter. Espera-se que após a dinâmica os alunos apresentem uma mudança de atitude, que eles reconheçam a importância do planejamento e respondam 
de forma planejada. A dinâmica é aplicada como atividade introdutória de disciplinas de gestão e gerenciamento de projetos, de modo a sensibilizar os alunos a importância do conteúdo da disciplina. Assim, não é necessário que os participantes tenham conhecimentos prévios em gerenciamento de projetos.

A dinâmica simula as fases de planejamento e execução do gerenciamento de projetos, onde os estudantes precisam planejar o tempo e os recursos (canetas, folhas de papel e fita adesiva) que precisarão para desenvolver o produto final, um Domo Geodésio. No início da atividade, os alunos, divididos em grupos de no máximo 10 participantes, recebem uma folha com as instruções do que precisam realizar na atividade. Em seguida, cada grupo deve definir um gerente de projetos, responsável por coordenar as atividades do grupo, planejar e estimar um tempo necessário para a construção do Domo Geodésio. Nos 30 minutos de planejamento, os alunos podem tirar suas dúvidas com o instrutor da atividade sem restrições, porém, na fase de execução, os alunos poderão fazer somente duas perguntas, por escrito, para o instrutor.

$\mathrm{Na}$ fase de execução os alunos seguem três etapas para construir tubos de papel e montar o Domo. Na primeira etapa, cada grupo deve construir 65 tubos de papel enrolados em diagonal, partindo de uma extremidade à outra: 35 tubos de 65 centímetros (tubo tamanho G) e 30 tubos de 58 centímetros (tamanho P). Na segunda etapa, cada grupo deve montar seis pentágonos. O passo inicial para montar um pentágono é montar um triângulo isósceles composto por 2 tubos $P$ e 1 tubo $G$, conforme apresenta a Figura 2a. Em seguida, deve-se acrescentar mais dois tubos (um G e um P), até formar o pentágono, conforme apresenta a Figura $2 b$.

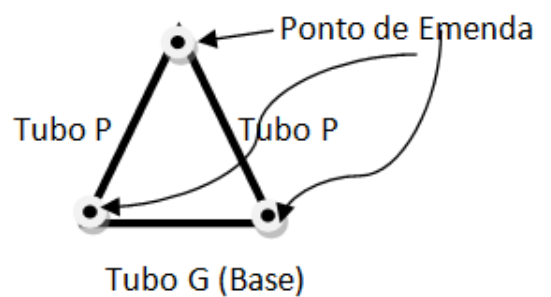

Figura 2a. Construindo triângulos isósceles

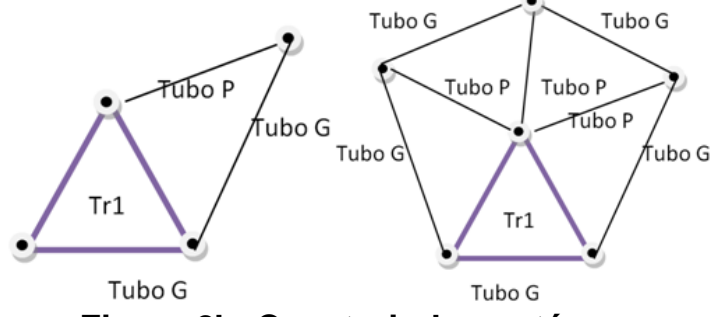

Figura 2b. Construindo pentágonos

A terceira etapa objetiva montar a estrutura do Domo com base nos pentágonos construídos na etapa anterior. São necessários 5 pentágonos para montar a base do Domo e mais 1 pentágono para montar o teto do Domo. Os pentágonos devem ser unidos conforme apresenta a Figura 3.

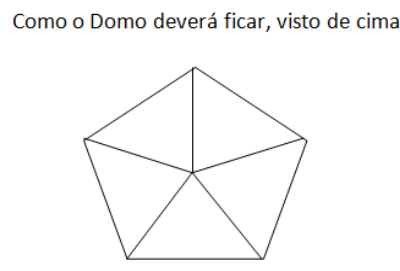

Pontos de emenda, que devem formar um círculo.

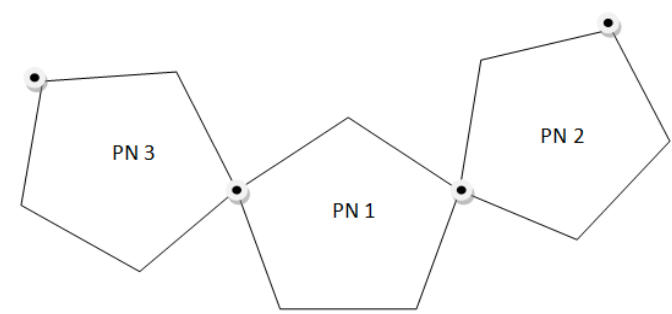

Figura 3. Montando a estrutura do Domo

Os cinco pentágonos devem ser colocados em círculos, unindo-os em apenas um ponto. Ao final, o sexto pentágono deverá fechar a parte de cima e os tubos que restam são usados para fechar a base da estrutura do Domo. 
Ao final da dinâmica, o instrutor coordena uma discussão com todos os participantes de modo a refletir sobre as lições aprendidas na atividade, as oportunidades de aprendizagem e o reconhecimento da importância do planejamento. Algumas questões contribuem na discussão, tais como: Como o grupo se planejou? $\mathrm{O}$ que deu certo no planejamento? O que deu errado? O planejamento ajudou na execução das atividades? Os participantes executaram as atividades como planejado? Qual a importância do planejamento para a execução das atividades no tempo disponível?

\section{Definição e Execução da Avaliação da Dinâmica PMDome}

O objetivo deste trabalho é analisar a dinâmica vivencial PMDome, com o propósito de avaliar a sua qualidade com respeito a experiência do jogador e percepção da aprendizagem sob o ponto de vista de alunos, no contexto de cursos de computação. A partir deste objetivo, seguindo o modelo MEEGA+ [Petri et al., 2017b] são definidas as seguintes questões de análise, agrupadas pelos fatores de qualidade avaliados:

\section{Experiência do Jogador}

QA1: A dinâmica PMDome proporciona uma experiência positiva aos jogadores?

QA2: Há diferença na experiência do jogador proporcionada aos alunos de nível técnico e especialização?

\section{Percepção da Aprendizagem}

QA3: A dinâmica PMDome contribui para a aprendizagem?

QA4: Há diferença na percepção da aprendizagem entre os alunos de nível técnico e especialização?

Foram realizados dois estudos de caso (Tabela 1) utilizando a dinâmica PMDome em duas turmas de computação, em diferentes níveis de ensino, em nível técnico e especialização. Em cada estudo de caso após o tratamento (aplicação da dinâmica PMDome), o questionário adaptado do modelo MEEGA+ foi respondido pelos participantes (alunos) para coletar os dados da avaliação da dinâmica PMDome. A pesquisa realizada foi aprovada pelo Comitê de Ética da Universidade Federal de Santa Catarina, instituição executora do estudo, sob parecer número 1.601.297 de 21/06/2016.

Tabela 1 - Estudos de caso realizados

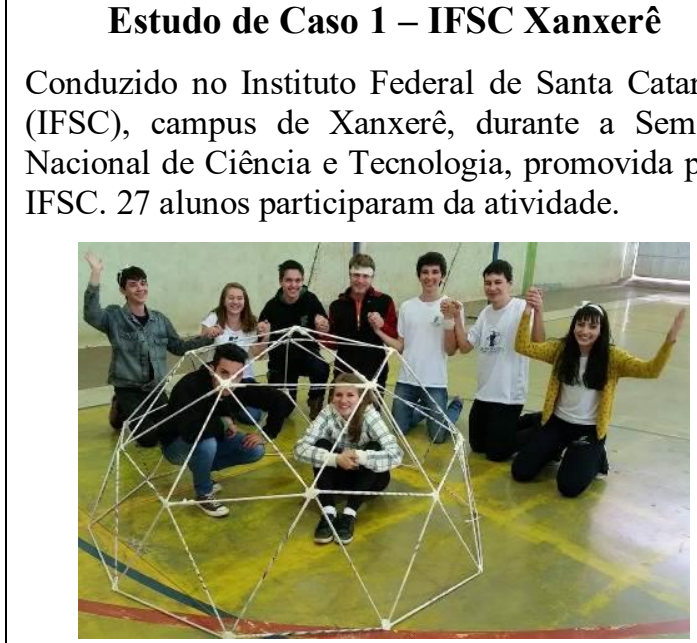

Figura 4. Estudo de caso 1 - IFSC Xanxerê

\section{Estudo de Caso 2 - IFFar Panambi}

Conduzido no Instituto Federal Farroupilha (IFFar), campus Panambi, na aula introdutória do curso de Especialização em Gestão de Tecnologia da Informação, com 20 alunos.

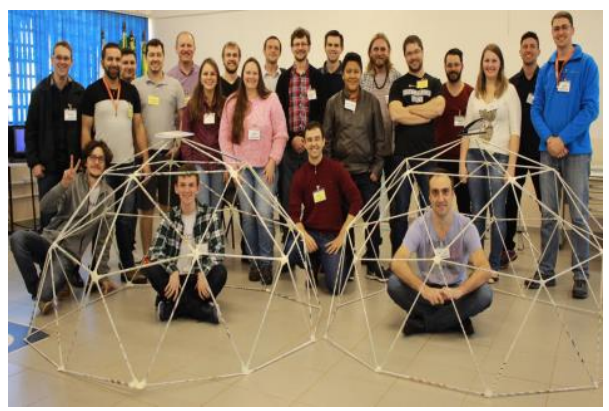

Figura 5. Estudo de caso 2 - IFFar Panambi 
No total participaram 47 alunos, 35 homens e 12 mulheres. A maioria dos alunos possui menos de 18 anos (27 alunos), 9 alunos possuem entre 18 e 28 anos, 7 alunos possuem de 29 a 39 anos e 4 alunos possuem mais de 40 anos. 34\% dos alunos costumam jogar semanalmente, $21 \%$ jogam mensalmente e $34 \%$ raramente jogam jogos não-digitais e/ou dinâmicas.

\section{Análise dos dados}

Os dados coletados nos dois estudos de caso foram agrupados em uma única amostra para avaliar a qualidade da dinâmica PMDome de modo a responder as questões de análise QA1 e QA3. No entanto, para analisar as questões de análise QA2 e QA4, os dados de cada estudo de caso são analisados separadamente, e são representados pela mediana das respostas para cada item avaliado.

\section{QA1: A dinâmica PMDome proporciona uma experiência positiva aos jogadores?}

De forma geral, em termos de experiência do jogador, os alunos perceberam uma positiva experiência proporcionada (Figura 6). Isso demonstra que o uso da dinâmica PMDome, como estratégia instrucional, proporcionou uma boa experiência aos jogadores, especialmente em termos de diversão, interação e cooperação entre os alunos.

Os alunos confirmaram que a dinâmica PMDome possui uma boa usabilidade, indicando que foi fácil de aprender e começar a jogar e, principalmente, que as regras eram claras e compreensíveis. A primeira impressão dos alunos foi de que a dinâmica não seria fácil para eles. No entanto, a organização do conteúdo envolvido na dinâmica contribuiu para que os alunos se sentissem confiantes de que aprenderiam com ela.

Os alunos avaliaram que a dinâmica foi desafiadora a eles, apresentando variações de desafios e/ou obstáculos em um ritmo adequado. No entanto, cabe salientar que alguns alunos (15\%) indicaram que a dinâmica se tornou monótona ou repetitiva. Esta avaliação pode estar relacionada ao momento de criação dos tubos de papel para a montagem do domo, pois é preciso construir 65 tubos e então, pode se tornar uma tarefa repetitiva.

PMDome também proporcionou um sentimento de satisfação e realização aos alunos. E o esforço coletivo foi importante para o grupo avançar na dinâmica. Este resultado também está relacionado a avaliação da interação social, que foi avaliada de forma bastante positiva. Isso demonstra que a dinâmica pode ser uma boa estratégia para promover a interação entre os alunos. Os alunos avaliaram que a dinâmica contribuiu para a cooperação e proporcionou uma experiência divertida com outras pessoas. Além disso, eles foram capazes de interagir com os colegas durante a dinâmica, proporcionando uma sensação de ambiente compartilhado.

Em relação à atenção focada, a dinâmica também foi avaliada de forma positiva. Este resultado mostra que os alunos tiveram uma experiência de envolvimento profundo na dinâmica, esquecendo-se de suas atividades diárias, concentrando-se nas tarefas da dinâmica e perdendo a noção do tempo durante a atividade. Os alunos indicaram que a dinâmica foi divertida e que algumas situações da dinâmica os fizeram sorrir. Os alunos também indicaram que a dinâmica foi relevante para seus interesses, indicando a relação da atividade com o conteúdo aprendido. Além disso, os alunos classificaram a dinâmica 
PMDome como um método de ensino adequado e que preferem aprender desta forma do que com outro método de ensino.

\section{QA2: Há diferença na experiência do jogador proporcionada aos alunos de nível técnico e especialização?}

Analisando a experiência proporcionada pela dinâmica PMDome nos diferentes níveis de ensino, identificou-se que ambas as turmas a avaliaram como positiva (Figura 6). Isto demonstra que, embora os interesses dos alunos e o foco dos cursos de nível técnico e especialização sejam diferentes, todos os alunos experimentaram a dinâmica PMDome como uma estratégia de ensino positiva e envolvente usando uma dinâmica vivencial. Em termos de usabilidade e diversão, ambas as turmas avaliaram de forma similar, indicando a dinâmica PMDome como uma experiência positiva.

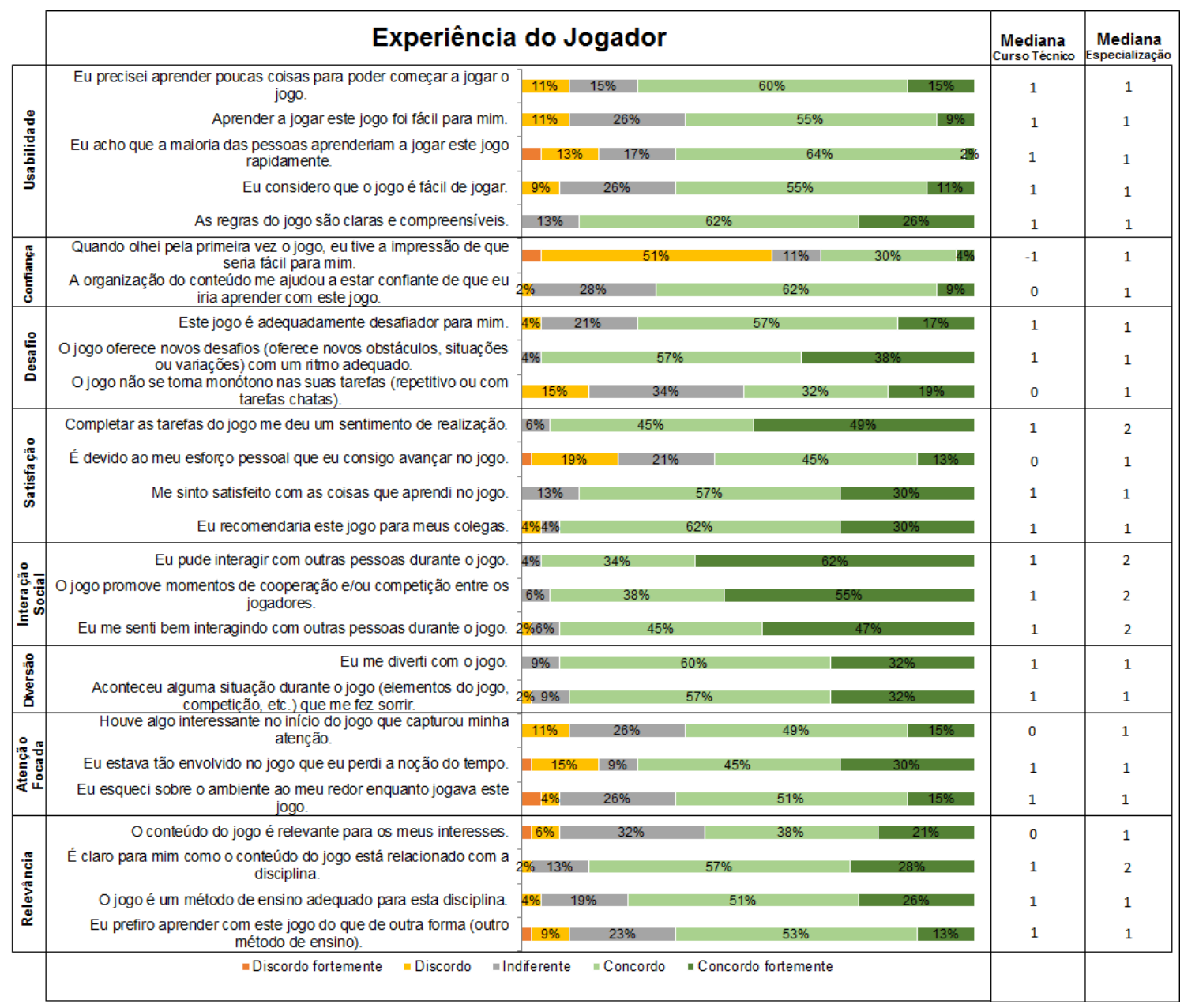

Figura 6. Diagrama de frequência e medianas da avaliação da experiência do jogador

Em termos de confiança, os alunos do curso de especialização sentiram-se mais confiantes de que a dinâmica seria fácil e que aprenderiam com ela, do que os alunos de nível técnico. Este resultado pode estar relacionado a maturidade e experiência dos alunos, pelo fato de que os alunos de nível técnico tiveram pouco acesso a informação sobre a área de gerenciamento de projetos durante o curso.

Em relação ao desafio proporcionado pela dinâmica PMDome, embora com resultado similar, os alunos do curso de especialização avaliaram que a dinâmica não se 
torna monótona, ao contrário dos alunos do curso técnico, que indicaram que a dinâmica se torna monótona ou com tarefas chatas. Esta avaliação pode estar relacionada as tarefas repetitivas de criação dos materiais (tubos) para a montagem do domo. E devido a dinamicidade do perfil dos alunos na faixa etária dos 17 anos, podem ter considerado esta tarefa repetitiva e/ou chata. O que evidencia a necessidade de desenvolver atividades bastante dinâmicas quando o público alvo de uma atividade possui este perfil.

A dinâmica proporcionou um sentimento de satisfação aos alunos de ambos os cursos, especialmente aos alunos do curso de especialização. De mesmo modo a interação social foi avaliada positiva por todos os alunos. No entanto, os alunos de especialização avaliaram de forma bastante positiva a interação e cooperação com os colegas durante a realização da dinâmica. Também estimulou a concentração e a atenção focada dos alunos, capturando a atenção dos alunos de especialização logo no início da atividade. Este resultado é importante, pelo fato de promover o engajamento dos alunos na atividade estimulando o pensamento, a criatividade e a interação social. Em termos de relevância, embora a dinâmica tenha sido indicada como relevante por ambas as turmas, os alunos de especialização a avaliaram de forma mais positiva, indicando a forte relação da dinâmica com o conteúdo envolvido.

\section{QA3: A dinâmica PMDome contribui para a aprendizagem?}

De forma geral, em termos de aprendizagem, os alunos perceberam uma positiva contribuição da dinâmica para a sua aprendizagem sobre gerenciamento de projetos (Figura 7). Os alunos indicaram uma percepção que a dinâmica PMDome contribuiu para a sua aprendizagem na disciplina; que a dinâmica foi eficiente quando comparado com outras estratégias de ensino utilizadas e também que a dinâmica contribuiu para os alunos reconhecerem a importância do gerenciamento para a realização de um projeto, incluindo o gerenciamento de materiais, tempo, recursos humanos, riscos, etc.

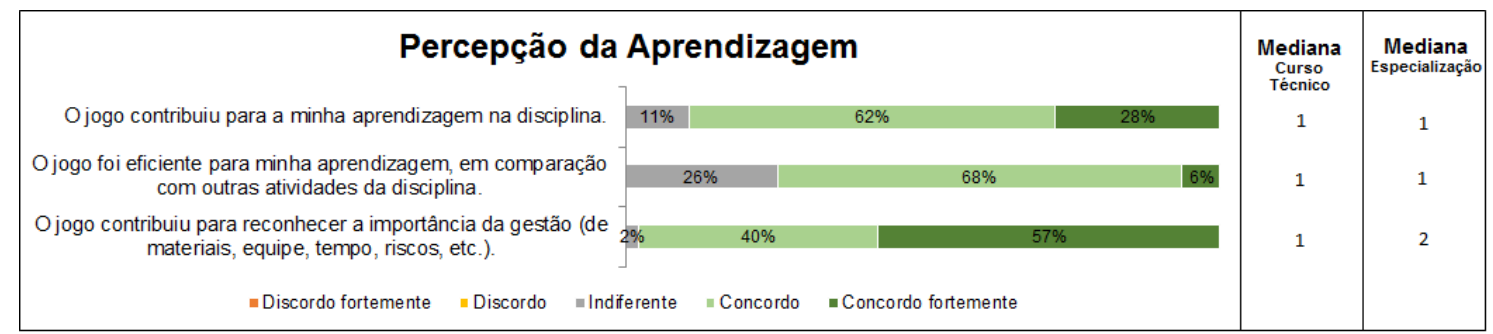

Figura 7. Diagrama de frequência e medianas da avaliação da percepção da aprendizagem

\section{QA4: Há diferença na percepção da aprendizagem entre os alunos de nível técnico e especialização?}

Analisando a avaliação da percepção da aprendizagem de cada turma (Figura 7), observou-se que, embora as avaliações são, no geral, positivas, os alunos dos cursos de especialização indicaram forte concordância ao avaliarem que a dinâmica PMDome contribuiu para reconhecer a importância do gerenciamento. Este resultado pode estar relacionado a experiência profissional e a visão sobre a área de computação que os alunos de especialização podem ter mais desenvolvido quando comparado aos alunos de nível técnico, iniciantes na área de computação. No entanto, é perceptível que a dinâmica PMDome contribuiu para a aprendizagem dos alunos, tanto de nível técnico, como os alunos de especialização. 


\subsection{Ameaças à validade}

Devido as características deste tipo de pesquisa, este trabalho está sujeito a algumas ameaças à validade. Deste modo, foram identificadas ameaças potenciais e aplicadas estratégias de mitigação para minimizar o impacto no trabalho. Para tratar as limitações na medição dos fatores de qualidade avaliados (conceitos subjetivos como experiência do jogador), foi usado o modelo MEEGA+ [Petri et al., 2017b] um modelo de avaliação de jogos sistematicamente desenvolvido e avaliado em termos de validade e confiabilidade [Petri et al., 2018]. Embora a pesquisa tenha envolvido um número aceitável de alunos de diferentes níveis de ensino, a aplicação da pesquisa nos próximos anos e em mais disciplinas pode ser uma alternativa para consolidar os resultados a partir deste trabalho. Além disso, como não houve um pré-teste medindo, por exemplo, o nível de conhecimento dos alunos antes da aplicação da dinâmica, pois causaria uma interrupção no fluxo das aulas, não foi possível identificar com exatidão o quanto cada aluno aprendeu sobre o assunto. No entanto, embora sem consenso, existem evidências de que a auto avaliação fornece informações confiáveis, válidas e úteis para este tipo de estudo [Sitzmann et al., 2010].

\section{Conclusão}

Com base em uma série de estudos de caso obteve-se uma primeira indicação que a dinâmica vivencial utilizada em workshops profissionais (PMDome), pode ser uma estratégia de ensino de gerenciamento de projetos em cursos de nível técnico e especialização com um impacto benéfico. Os resultados evidenciam a qualidade da dinâmica PMDome para a aprendizagem dos 47 alunos participantes de nível técnico e especialização. Além de contribuir positivamente para a interação social dos alunos, diversão, satisfação e confiança.

Portanto, conclui-se que a utilização de dinâmicas utilizadas em workshops profissionais, em especial a dinâmica PMDome, possui também um forte potencial em contribuir para a aprendizagem dos alunos também quando inserida em contexto educacional. Como trabalhos futuros, pretende-se aplicar e avaliar a dinâmica PMDome em outros níveis de ensino de modo a confirmar os resultados e identificar oportunidades de melhoria.

\section{Agradecimentos}

Aos alunos do Curso Técnico em Informática do IFSC e da Especialização em Gestão de Tecnologia da Informação do IFFar, pela participação nos estudos de caso.

Este trabalho foi apoiado pelo $\mathrm{CNPq}$ (Conselho Nacional de Desenvolvimento

Científico e Tecnológico), uma entidade do governo brasileiro focada no desenvolvimento científico e tecnológico.

\section{Referências}

ACM/IEEE-CS. (2013). Computer Science Curricula 2013: Curriculum Guidelines for Undergraduate Degree Programs in Computer Science.

Barnes, T., Powell, E., Chaffin, A., e Lipford, H. (2008). Game2Learn: improving the motivation of CS1 students. Proc. of the 3rd Int. Conf. on Game Development in Computer Science Education, (pp. 1-5). New York, NY, USA. 
Basili, V. R., Caldiera, G., e Rombach, H. D. (1994). Goal, Question Metric Paradigm. In J. J. Marciniak, Encyclopedia of Software Engineering. New York, USA.

Battistella, P., Camargo, A., Gresse von Wangenheim, C. (2016). SCRUM-Scape: Jogo educacional de Role-Playing Game (RPG) para ensinar SCRUM. $27^{\circ}$ Simpósio Brasileiro de Informática na Educação, (pp. 330-339). Uberlândia, MG, Brasil.

Calderón A. e Ruiz M. (2015). A systematic literature review on serious games evaluation: An application to software project management. Computers \& Education, vol. 87, 396-422.

Çiftci, S. (2018). Trends of Serious Games Research from 2007 to 2017: A Bibliometric Analysis. Journal of Education and Training Studies, 6(2), 18-27.

Connolly, T. M., Boyle, E. A., MacArthur, E., Hainey, T., \& Boyle, J. M. (2012). A systematic literature review of empirical evidence on computer games and serious games. Computers \& Education, 59(2), 661-686.

Gibson, B. \& Bell, T. (2013). Evaluation of games for teaching computer science. Proc. of the 8th Workshop in Primary and Secondary Computing Education, (pp. 51-60). ACM, New York, NY, USA.

Gresse von Wangenheim, C., Savi, R., e Borgatto, A. F. (2013). SCRUMIA - An educational game for teaching SCRUM in computing courses. Journal of Systems and Software, 86(10), 2675-2687.

Kosa, M., Yilmaz, M., O'Connor, R., \& Clarke, P. (2016). Software engineering education and games: a systematic literature review. Journal of Universal Computer Science, 22(12), 1558-1574.

Letra, P., Paiva, A. C. R., \& Flores, N. (2015). Game Design Techniques for Software Engineering Management Education. Proc. of the 18th Int. Conf. on Computational Science and Engineering, (pp. 192-199). IEEE, Porto, Portugal.

Parsons, P. (2011). Preparing computer science graduates for the 21st Century. Teaching Innovation Projects, 1(1), article 8.

Paasivaara, M., Heikkilä, V., Lassenius, C. \& Toivola, T. (2014). Teaching students Scrum using LEGO blocks. Proc. of the 36th Int. Conf. on Software Engineering, (pp. 382-391). ACM, New York, NY, USA.

Petri, G. e Gresse von Wangenheim, C. (2016). How to Evaluate Educational Games: a Systematic Literature Review. Journal of Universal Computer Science, 22(7), 9921021.

Petri, G. e Gresse von Wangenheim, C. (2017). How games for computing education are evaluated: a systematic literature review. Computers \& Education, vol. 107, 6890 .

Petri, G., Gresse von Wangenheim, C., e Borgatto, A. F. (2017b). Evolução de um Modelo de Avaliação de Jogos para o Ensino de Computação. $25^{\circ}$ Workshop sobre Educação em Computação, (pp. 2327-2336). São Paulo, SP, Brasil.

Petri, G., Gresse von Wangenheim, C., e Borgatto, A. F. (2017a). Quality of Games for Teaching Software Engineering: An Analysis of Empirical Evidences of Digital and Non-digital Games. Proc. of the 39th Int. Conf. on Software Engineering: Software 
Engineering Education and Training Track, (pp. 150-159). IEEE/ACM, Buenos Aires, Argentina.

Petri, G., Gresse von Wangenheim, C., e Borgatto, A. F. (2018). MEEGA+, Systematic Model to Evaluate Educational Games. In Newton Lee (Eds) Encyclopedia of Computer Graphics and Games, (pp. 1-7). Cham: Springer.

Petri, G., Battistella, P., Cassettari, F., Gresse von Wangenheim, C., e Hauck, J. (2016). Um Quiz Game para a revisão de conhecimento em Gerenciamento de Projetos. $27^{\circ}$ Simpósio Brasileiro de Informática na Educação, (pp. 320-329). Uberlândia, MG, Brasil.

Petri, G. e Marcon Júnior, R. P. (2014). Um jogo educacional para o ensino de metodologias ágeis. $7^{\circ}$ Fórum de Educação em Engenharia de Software, (pp. 66-69). Maceió, AL, Brasil.

PMDome. (2017). PMDome Workshop. Disponível em: <https://ricardovargas.com/pt/workshops/pmdome/> Acesso em: 27 novembro 2017.

Sitzmann, T., Ely, K., Brown, K. G., e Bauer, K. N. (2010). Self-Assessment of Knowledge: A Cognitive Learning or Affective Measure? Academy of Management Learning \& Education, 9(2), 169-191.

Souza, M. e França, C. (2016). O que explica o sucesso de jogos no ensino de engenharia de software? Uma teoria de motivação. $24^{\circ}$ Workshop sobre Educação em Computação, (pp. 2255-2263). Porto Alegre, RS, Brasil.

Standish Group. (2015). CHAOS Report 2015.

Yin, R.K. (2017). Case study research and applications: design and methods (5 ed.). Sage Publications, Beverly Hills. 